\title{
Structural Damping Effects on Dynamic Instability of Subtangentially Loaded and Shear Deformable Beck's Columns
}

\author{
Dong-Ju Min, ${ }^{1}$ Jaegyun Park, ${ }^{2}$ Sang-Ho Yeon, ${ }^{3}$ and Moon-Young Kim ${ }^{1}$ \\ ${ }^{1}$ School of Civil and Architectural Engineering, Sungkyunkwan University, 2066 Seobu-ro, Jangan-gu, \\ Suwon-si 16419, Republic of Korea \\ ${ }^{2}$ Department of Civil \& Environmental Engineering, Dankook University, 126 Jukjeon-dong, Suji-gu, \\ Yongin-si 448-701, Republic of Korea \\ ${ }^{3}$ Department of Civil Engineering, Semyung University, 579 Sinwoul-dong, Jecheon-si, Chungbuk 390-711, Republic of Korea
}

Correspondence should be addressed to Moon-Young Kim; kmye@skku.edu

Received 14 April 2016; Revised 10 June 2016; Accepted 12 June 2016

Academic Editor: Anders Eriksson

Copyright (C) 2016 Dong-Ju Min et al. This is an open access article distributed under the Creative Commons Attribution License, which permits unrestricted use, distribution, and reproduction in any medium, provided the original work is properly cited.

\begin{abstract}
A frequency equation of externally and internally damped and shear-flexible cantilever columns subjected to a subtangentially follower force is analytically derived in a dimensionless form with relation to the linear instability theory of Beck's columns. Some parametric studies are then performed with variation of two damping coefficients under the assumption of Rayleigh damping. Based on the analysis results, it is demonstrated that three damping cases in association with flutter loads of Beck's columns can be selected including one case representative of structural damping. Finally, stability maps of shear-flexible and damped Beck's columns are constructed for the three damping cases and discussed in the practical range of damping coefficients and shear parameters. In addition, flutter loads and time history analysis results are presented using dimensionless FE analysis and compared with exact solutions.
\end{abstract}

\section{Introduction}

The dynamic instability problem of a cantilever column subjected to a follower force has been well known and various interesting related topics have been intensively studied by many researchers since Beck [1] solved this problem analytically. It is worth referring to the monographs by Ziegler [2], Bolotin [3], and Leipholz [4] who addressed the static and dynamic stability of a nonconservative system.

According to the linear stability theory of nonconservative systems, external damping tends to increase flutter loads but columns under small internal damping lose their stability at the flutter load drastically deceased. In this case, Beck's columns subjected to follower forces slightly larger than internally damped flutter loads become unstable in form of oscillations with a slow growth of amplitude, which is sometimes called a quiet flutter. This destabilizing effect of small internal damping on the stability of nonconservative systems, Ziegler's paradox, has been one of attractive research topics [5-9]. Particularly, Langthjem and Sugiyama [10] and Elishakoff [11] published survey papers regarding the dynamic stability of columns subjected to follower loads.

The effects of shear deformation and rotary inertia on the stability of Beck's columns have been investigated by many scholars [12-19]. Most have investigated shear effects on the divergence and flutter loads of nonconservative systems using FE formulations, but those studies neglected the effects of damping. Some researchers [20-27] noticed that external and internal (viscoelastic) damping of nonconservative systems can be treated as Rayleigh damping and explored the destabilizing effects of internal damping using an analytical approach or FE analysis.

To the authors' knowledge, it is judged that a closedform solution of Beck's problem considering not only internal 


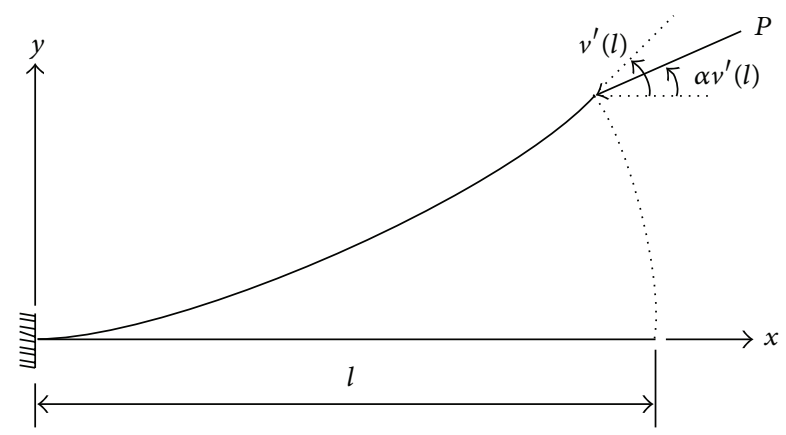

Figure 1: Beck's column under a subtangential follower force.

and external damping effects but also the effects of shear deformation and rotary inertia has not been proposed to date, and its stability behavior in the practical range of internal and external damping has not been reported. The important points presented in this paper are summarized as follows:

(1) A linear stability theory of subtangentially loaded, damped, and shear-flexible Beck's columns is first formulated in a dimensionless form.

(2) A frequency equation and a buckling equation of Timoshenko cantilever beams subjected to both a subtangentially follow force and internal and external damping forces are then derived in the closed form to determine the damped flutter load and divergence load, respectively.

(3) Based on the well-known fact that internal and external damping can be transformed to Rayleigh damping, it is shown that the damping coefficients can be effectively determined using proportional damping and two damping coefficients can be reasonably estimated for real systems through a parametric study of damping coefficients in association with flutter loads of Beck's columns.

(4) Finally, stability maps of shear deformable and damped Beck columns are newly constructed using the analytical solution, which is compared with the FE solution in the practical range of damping coefficients and shear parameters.

\section{Analytical Formulation for Instability Theory of Damped Beck's Columns}

In this section, a linear stability theory of damped and shear deformable Beck's columns is formulated using an analytical approach and its frequency equation is derived in the closed form.

2.1. Stability Theory of Damped and Shear Deformable Beck's Columns. Figure 1 shows a prismatic cantilever column subjected to a subtangential follower force $P$ at the tip end $A$ in which the direction of the force changes subtangentially according to the deformed column axis. The coordinate $x$ is measured along the centroidal axis of the column and the cross-section properties are constant throughout the column length.

The extended Hamilton's principle for shear deformable Beck's columns including external and internal damping forces and the nonconservative parameter $\alpha$ can be expressed as

$$
\begin{aligned}
& \int_{t_{1}}^{t_{2}}\left[\int_{o}^{l}\left\{\rho A\left(\dot{v} \delta \dot{v}+\frac{l^{2} \dot{\beta} \delta \dot{\beta}}{\lambda^{2}}\right)-E I\left(\beta^{\prime} \delta \beta^{\prime}+\frac{\left(v^{\prime}-\beta\right) \delta\left(v^{\prime}-\beta\right)}{S l^{2}}\right)+P v^{\prime} \delta v^{\prime}\right\} d x\right. \\
& \left.-\int_{o}^{l}\left\{\gamma_{1}\left(\dot{v} \delta v+\frac{l^{2} \dot{\beta} \delta \beta}{\lambda^{2}}\right)+\gamma_{2}\left(\dot{\beta}^{\prime} \delta \beta^{\prime}+\frac{\left(\dot{v}^{\prime}-\dot{\beta}\right) \delta\left(v^{\prime}-\beta\right)}{S l^{2}}\right)\right\} d x-\alpha P v^{\prime}(l) \delta v(l)\right] d t=0,
\end{aligned}
$$

where $l$ is the total length of the column; $v, \beta$ is the lateral displacement of the column and the bending rotation of its cross section; $A, A_{s}$ are the sectional area and the effective shear area; $\rho A$ is the mass per unit length; $E I, G A_{s}$ are the flexural and the shear rigidity; $\lambda(\equiv l / \sqrt{I / A})$ is slenderness ratio; $S\left(\equiv E I /\left(G A_{s} l^{2}\right)\right)$ is dimensionless shear parameter; $\gamma_{1}$ and $\gamma_{2}$ are the external and internal (viscoelastic) damping coefficients, respectively; $\alpha$ is the nonconservativeness parameter denoting subtangentiality; the shear angle is related to the bending angle by $\varphi=v^{\prime}-\beta$.

The first three terms in the square brackets in (1) are the kinetic energy, the elastic strain energy of the system, and the potential energy due to the axial force $P$, respectively; and the fourth, fifth, and last terms denote the works done by nonconservative damping and the follower end force. Also, the symbol $\delta$ denotes the first variation, $t$ represents time, and $t_{1}$ and $t_{2}$ are the integration limits.

For convenience, the following dimensionless variables including $\lambda$ and $S$ are introduced:

$$
\begin{aligned}
& v^{*}=\frac{v}{l}, \\
& \beta^{*}=\beta, \\
& x^{*}=\frac{x}{l}, \\
& t^{*}=\frac{t \sqrt{E I /(\rho A)}}{l^{2}},
\end{aligned}
$$




$$
\begin{aligned}
& P^{*}=\frac{P l^{2}}{E I}, \\
& \gamma_{1}^{*}=\frac{\gamma_{1} l^{2}}{\sqrt{\rho A E I}},
\end{aligned}
$$

$$
\begin{aligned}
& \int_{t_{1}^{*}}^{t_{i}}\left[\int_{0}^{1}\left\{\left(i^{*} \delta \dot{v}^{*}+\frac{\dot{\beta}^{*} \delta \dot{\beta}^{*}}{\lambda^{2}}\right)-\left(\beta^{* \prime} \delta \beta^{* \prime}+\frac{\left(v^{* \prime}-\beta\right) \delta\left(v^{* \prime}-\beta\right)}{S}\right)+P^{*} v^{* \prime} \delta v^{* \prime}-\gamma_{1}^{*}\left(\dot{v}^{*} \delta v^{*}+\frac{\dot{\beta}^{*} \delta \beta^{*}}{\lambda^{2}}\right)-\gamma_{2}^{*}\left(\dot{\beta}^{* \prime} \delta \beta^{* \prime}+\frac{\left(\dot{i}^{* \prime}-\dot{\beta}^{*}\right) \delta\left(v^{* \prime}-\beta^{*}\right)}{S}\right)\right\} d x^{*}\right. \\
& \left.-\alpha P^{*} v^{* \prime}(1) \delta v^{*}(1)\right] d t^{*}=0 .
\end{aligned}
$$

Now invoking Hamilton's principle leads to the following dimensionless equation of motion and boundary conditions:

$$
\begin{gathered}
\ddot{v}^{*}-\frac{1}{S}\left(\dot{v}^{* \prime \prime}-\beta^{* \prime}\right)+\left(P^{*} v^{* \prime}\right)^{\prime}+\gamma_{1}^{*} \dot{v} \\
-\frac{\gamma_{2}^{*}\left(\dot{v}^{* \prime \prime}-\dot{\beta}^{* \prime}\right)}{S}=0, \\
\beta^{* \prime \prime}-\frac{\ddot{\beta}^{*}}{\lambda^{2}}+\frac{1}{S}\left(v^{* \prime}-\beta^{*}\right)-\frac{\gamma_{1}^{*} \dot{\beta}^{*}}{\lambda^{2}} \\
+\gamma_{2}^{*}\left(\dot{\beta}^{* \prime \prime}+\frac{\dot{v}^{* \prime}-\dot{\beta}^{*}}{S}\right)=0
\end{gathered}
$$

in which the geometric and natural boundary conditions are

$$
\begin{aligned}
& \delta v^{*}(0)=0, \\
& \delta \beta^{*}(0)=0, \\
& \beta^{* \prime}(1)+\gamma_{2}^{*} \dot{\beta}^{* \prime}(1)=0, \\
& v^{* \prime}(1)-\beta^{*}(1)+\gamma_{2}^{*}\left(\dot{v}^{* \prime}(1)-\dot{\beta}^{*}(1)\right) \\
& \quad-(1-\alpha) S P^{*}(1) v^{* \prime}(1)=0 .
\end{aligned}
$$

Hamilton's principle of Beck's columns considering shear deformation and rotary inertia based on Engesser's buckling theory has been presented by Attard et al. [19] in which damping effects were neglected. Recently it is worth noting that Elishakoff et al. [28] have discussed Timoshenko beam theory from a historical perspective.

\subsection{Frequency Equation of Damped and Shear Deformable} Beck's Columns. In order to analytically derive the frequency

$$
\begin{aligned}
& \lambda_{1}=\sqrt{a_{1}+\sqrt{b_{1}+a_{1}^{2}}}, \\
& \lambda_{2}=\sqrt{-a_{1}+\sqrt{b_{1}+a_{1}^{2}}},
\end{aligned}
$$

equation of Beck's columns, the vibrating mode shape may be assumed to be harmonic. Then substituting $v^{*}=e^{\Omega t^{*}} V\left(x^{*}\right)$ and $\beta^{*}=e^{\Omega t^{*}} B\left(x^{*}\right)$ into (4a) and (4b) and eliminating $B$ lead to

$$
\begin{aligned}
& \left(1+\Omega \gamma_{2}-P^{*} S\right)\left(1+\Omega \gamma_{2}\right) V^{\prime \prime \prime \prime} \\
& +\left[P^{*}\left\{1+\frac{\Omega^{2} S}{\lambda^{2}}+\Omega\left(\gamma_{2}+\frac{\gamma_{1} S}{\lambda^{2}}\right)\right\}\right. \\
& \left.-\Omega\left(\Omega+\gamma_{1}\right)\left(1+\Omega \gamma_{2}\right)\left(S+\frac{1}{\lambda^{2}}\right)\right] V^{\prime \prime}+\Omega(\Omega \\
& \left.+\gamma_{1}\right)\left\{1+\Omega \gamma_{2}+\frac{\Omega\left(\Omega+\gamma_{1}\right) S}{\lambda^{2}}\right\} V=0,
\end{aligned}
$$

where the dimensionless frequency $\Omega$ can be a complex number defined as

$$
\Omega\left(\equiv \eta^{*}+i \omega^{*}\right)=(\eta+i \omega) l^{2} \sqrt{\frac{\rho A}{E I}}
$$

in which $\eta$ and $\omega$ are the real and imaginary parts of the frequency.

Finally the general solution to (5) is obtained with consideration of (4a) and (4b) as

$$
\begin{aligned}
V\left(x^{*}\right)= & A \cosh \left(\lambda_{1} x^{*}\right)+B \sinh \left(\lambda_{1} x^{*}\right) \\
& +C \cos \left(\lambda_{2} x^{*}\right)+D \sin \left(\lambda_{2} x^{*}\right), \\
B^{\prime}\left(x^{*}\right)= & \frac{1-S P^{*}+\gamma_{2}^{*} \Omega}{1+\gamma_{2}^{*} \Omega} V^{\prime \prime}-\frac{S \Omega}{1+\gamma_{2}^{*} \Omega}\left(\Omega+\gamma_{1}^{*}\right) V,
\end{aligned}
$$

where

Equation (1) can be then rewritten using (2a), (2b), (2c), (2d), in 


$$
\begin{aligned}
& a_{1}=\frac{\Omega\left(\Omega+\gamma_{1}\right)\left(1+\Omega \gamma_{2}\right)\left(S+1 / \lambda^{2}\right)-P^{*}\left\{1+\Omega \gamma_{2}+\Omega S\left(\Omega+\gamma_{1}\right) / \lambda^{2}\right\}}{2\left(1+\Omega \gamma_{2}\right)\left(1+\Omega \gamma_{2}-P^{*} S\right)}, \\
& b_{1}=-\frac{\Omega\left(\Omega+\gamma_{1}\right)\left\{1+\Omega \gamma_{2}+\Omega S\left(\Omega+\gamma_{1}\right) / \lambda^{2}\right\}}{\left(1+\Omega \gamma_{2}\right)\left(1+\Omega \gamma_{2}-P^{*} S\right)} .
\end{aligned}
$$

In contrast, the boundary conditions of (4c)-(4f) can be rewritten with respect to $V, B^{\prime}$ as follows:

$$
\begin{aligned}
& V(0)=0, \\
& S B^{\prime \prime}(0)+\left(1+\gamma_{2}^{*}\right) V^{\prime}(0)=0, \\
& B^{\prime}(1)=0, \\
& \frac{1}{\lambda^{2}}\left\{\Omega^{2}+\gamma_{1} \Omega\right. \\
& \left.\quad-\frac{1-\alpha}{1+\gamma_{2} \Omega}\left(\frac{1}{\lambda^{2}}+S \gamma_{1} \Omega+S \Omega^{2}+\frac{\gamma_{2} \Omega}{\lambda^{2}}\right)(1-\alpha) P^{*}\right\} \\
& \quad \cdot V^{\prime}(1)-\left(1+\gamma_{2} \Omega\right) B^{\prime \prime}(1)=0 .
\end{aligned}
$$

Substituting (7a), (7b), (7c), (7d), (7e), and (7f) into (8) and invoking the four boundary conditions, a set of homogeneous equations with respect to $A, B, C$, and $D$ is obtained. Now for a nontrivial solution to exist, the determinant of the homogeneous equation must be zero. Finally we obtain a frequency equation of shear-flexible and damped Beck's columns subjected to a subtangential follower force in the analytical form as

$$
\begin{aligned}
& \lambda_{1}\left\{1+\lambda_{1}^{2} S-\frac{\left.\left\{P^{*} \lambda_{1}^{2}+\Omega\left(\Omega+\gamma_{1}^{*}\right)\right\} S^{2}\right\}}{1+\Omega \gamma_{2}^{*}}\right\}\left[\lambda _ { 2 } \left\{\lambda_{1}^{2}(1\right.\right. \\
& \left.\left.+\Omega \gamma_{2}^{*}-P^{*} S\right)-\Omega\left(\Omega+\gamma_{1}^{*}\right) S\right\} c_{2} \cos \left(\lambda_{2}\right) \cosh \left(\lambda_{1}\right) \\
& +\lambda_{1}\left\{\lambda_{2}^{2}\left(1+\Omega \gamma_{2}^{*}-P^{*} S\right)+\Omega\left(\Omega+\gamma_{1}^{*}\right) S\right\} c_{1} \\
& \quad \cdot \sin \left(\lambda_{2}\right) \sinh \left(\lambda_{1}\right)+\lambda_{2}\left\{\lambda_{2}^{2}\left(1+\Omega \gamma_{2}^{*}-P^{*} S\right)\right. \\
& \left.\left.+\Omega\left(\Omega+\gamma_{1}^{*}\right) S\right\} c_{2}\right]=\lambda_{2}\left\{1-\lambda_{2}^{2} S\right. \\
& \left.\quad+\frac{\left\{P^{*} \lambda_{2}^{2}-\Omega\left(\Omega+\gamma_{1}^{*}\right)\right\} S^{2}}{1+\Omega \gamma_{2}^{*}}\right\}\left[\lambda _ { 1 } \left\{\lambda _ { 2 } ^ { 2 } \left(1+\Omega \gamma_{2}^{*}\right.\right.\right. \\
& \left.\left.\quad-P^{*} S\right)+\Omega\left(\Omega+\gamma_{1}^{*}\right) S\right\} c_{1} \cos \left(\lambda_{2}\right) \cosh \left(\lambda_{1}\right) \\
& \quad-\lambda_{2}\left\{\lambda_{1}^{2}\left(1+\Omega \gamma_{2}^{*}-P^{*} S\right)-\Omega\left(\Omega+\gamma_{1}^{*}\right) S\right\} c_{2} \\
& \quad+\sin \left(\lambda_{2}\right) \sinh \left(\lambda_{1}\right) \\
& \left.+\lambda_{1}\left\{\lambda_{1}^{2}\left(1+\Omega \gamma_{2}^{*}-P^{*} S\right)-\Omega\left(\Omega+\gamma_{1}^{*}\right) S\right\} c_{1}\right]
\end{aligned}
$$

where

$$
\begin{aligned}
c_{1}= & {\left[(\alpha-1)\left(1+\Omega \gamma_{2}^{*}\right) P^{*}\right.} \\
& +P^{*}\left\{\frac{(\alpha-1) \Omega\left(\Omega+\gamma_{1}^{*}\right)}{\lambda^{2}}+\lambda_{1}^{2}\left(1+\Omega \gamma_{2}^{*}\right)\right\} S \\
& -\left(1+\Omega \gamma_{2}^{*}\right) \\
& \left.\cdot\left\{\lambda_{1}^{2}\left(1+\Omega \gamma_{2}^{*}\right)-\Omega\left(\Omega+\gamma_{1}^{*}\right)\left(S+\frac{1}{\lambda^{2}}\right)\right\}\right], \\
c_{2}= & {\left[(\alpha-1)\left(1+\Omega \gamma_{2}^{*}\right) P^{*}\right.} \\
& +P^{*}\left\{\frac{(\alpha-1) \Omega\left(\Omega+\gamma_{1}^{*}\right)}{\lambda^{2}}-\lambda_{2}^{2}\left(1+\Omega \gamma_{2}^{*}\right)\right\} S \\
& +\left(1+\Omega \gamma_{2}^{*}\right) \\
& \left.\cdot\left\{\lambda_{2}^{2}\left(1+\Omega \gamma_{2}^{*}\right)+\Omega\left(\Omega+\gamma_{1}^{*}\right)\left(S+\frac{1}{\lambda^{2}}\right)\right\}\right] .
\end{aligned}
$$

In particular, if both shear deformation and rotary inertia effects are neglected $(S=0.0, \lambda=\infty),(9)$ is reduced to

$$
\begin{aligned}
& \left(1+\Omega \gamma_{2}^{*}\right)\left(\lambda_{1}^{4}+\lambda_{2}^{4}\right)+P^{*}(1-\alpha)\left(\lambda_{1}^{2}-\lambda_{2}^{2}\right) \\
& +\left\{2 \lambda_{1}^{2} \lambda_{2}^{2}\left(1+\Omega \gamma_{2}^{*}\right)+P^{*}(\alpha-1)\left(\lambda_{1}^{2}-\lambda_{2}^{2}\right)\right\} \\
& \quad \cdot \cos \left(\lambda_{2}\right) \cosh \left(\lambda_{1}\right) \\
& +\lambda_{1} \lambda_{2}\left\{2 P^{*}(\alpha-1)+\left(\lambda_{2}^{2}-\lambda_{1}^{2}\right)\left(1+\Omega \gamma_{2}^{*}\right)\right\} \\
& \quad \cdot \sin \left(\lambda_{2}\right) \sinh \left(\lambda_{1}\right)=0 .
\end{aligned}
$$

In contrast, the frequency is zero in the case of a static divergence system. So the solution to (5) is

$$
\begin{aligned}
V\left(x^{*}\right)= & A \cos \left(\sqrt{\frac{P^{*}}{1-P^{*} S}} x^{*}\right) \\
& +B \sin \left(\sqrt{\frac{P^{*}}{1-P^{*} S}} x^{*}\right)+C x^{*}+D
\end{aligned}
$$

in which the divergence loads can be evaluated from the following buckling equation:

$$
\alpha+(1-\alpha)\left(1-P^{*} S\right) \cos \sqrt{\frac{P^{*}}{1-P^{*} S}}=0 .
$$




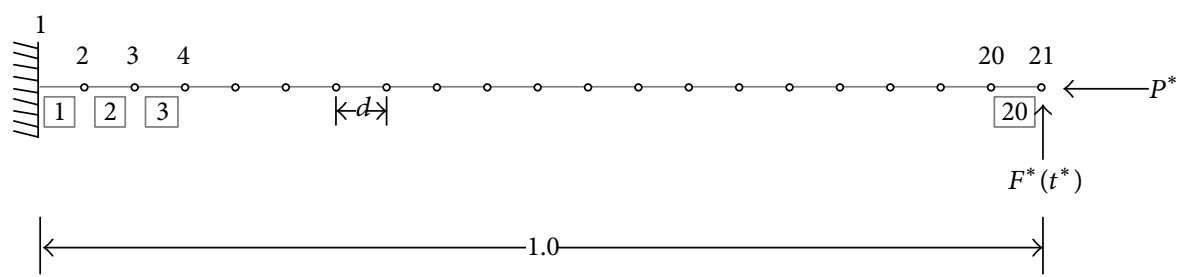

FIGURE 2: FE modeling of Beck's column subjected to a follower force and a lateral impulse load.

Detinko [29] presented a generalized version of (11) considering additionally the effects of a tip mass but neglected shear effects. However, to the authors' knowledge, the frequency equation (9) and the buckling equation (13) of shearflexible, subtangentially loaded, and internally and externally damped Beck's columns have first been derived in the closed form.

The critical flutter and divergence loads can be determined by constructing double eigencurves of $P^{*}-\Omega^{2}$ based on (9). Generally, the stability of Beck's columns depends on the location of $\Omega$ on the complex plane. As the follower force $P^{*}$ is increased, the system is stable if $\Omega$ stays in the lefthand half-plane $(\eta \leq 0)$. In an undamped nonconservative system, $\Omega$ remains on the pure imaginary axis $(\eta=0)$ at first, but the two frequencies coalesce and the real part $\eta$ changes negatively around the undamped flutter load $P_{\mathrm{fl}}^{*}$. The flutter loads $P_{\text {fl } d}^{*}$ of the damped system are calculated when $\eta$ transits from negative to positive values with the oscillatory part $\omega$ being nonzero. On the other hand, static divergence occurs when $\eta$ becomes positive and $\omega$ is equal to zero. Therefore, the divergence loads $P_{d}^{*}$ can be directly calculated from the root of (13).

Later it is required to analytically evaluate the natural frequencies of a shear-flexible cantilever beam without both damping forces and follower forces. To this end, (7c) and (7d) are simplified as

$$
\begin{aligned}
& \lambda_{1} \\
& =\sqrt{\Omega \sqrt{\left(\frac{\Omega\left(S+1 / \lambda^{2}\right)}{2}\right)^{2}-\left(1+\frac{\Omega^{2} S}{\lambda^{2}}\right)}+\frac{\Omega^{2}\left(S+1 / \lambda^{2}\right)}{2},} \\
& \lambda_{2} \\
& =\sqrt{\Omega \sqrt{\left(\frac{\Omega\left(S+1 / \lambda^{2}\right)}{2}\right)^{2}-\left(1+\frac{\Omega^{2} S}{\lambda^{2}}\right)}-\frac{\Omega^{2}\left(S+1 / \lambda^{2}\right)}{2} .}
\end{aligned}
$$

The resulting frequency equation for free vibration of the shear-flexible cantilever is

$$
\begin{gathered}
\lambda_{1}\left\{1+S\left(\lambda_{1}^{2}-\Omega^{2} S\right)\right\}\left[\lambda _ { 2 } ( \lambda _ { 1 } ^ { 2 } - \Omega ^ { 2 } S ) \left\{\lambda_{2}^{2}\right.\right. \\
\left.+\Omega^{2}\left(S+\frac{1}{\lambda^{2}}\right)\right\} \cos \left(\lambda_{2}\right) \cosh \left(\lambda_{1}\right)-\lambda_{1}\left(\lambda_{2}^{2}\right. \\
\left.+\Omega^{2} S\right)\left\{\lambda_{1}^{2}-\Omega^{2}\left(S+\frac{1}{\lambda^{2}}\right)\right\} \sin \left(\lambda_{2}\right) \sinh \left(\lambda_{1}\right)
\end{gathered}
$$

$$
\begin{aligned}
& \left.+\lambda_{2}\left(\lambda_{2}^{2}+\Omega^{2} S\right)\left\{\lambda_{2}^{2}+\Omega^{2}\left(S+\frac{1}{\lambda^{2}}\right)\right\}\right]+\lambda_{2}\{1 \\
& \left.-S\left(\lambda_{2}^{2}+\Omega^{2} S\right)\right\}\left[\lambda _ { 1 } ( \lambda _ { 2 } ^ { 2 } + \Omega ^ { 2 } S ) \left\{\lambda_{1}^{2}\right.\right. \\
& \left.-\Omega^{2}\left(S+\frac{1}{\lambda^{2}}\right)\right\} \cos \left(\lambda_{2}\right) \cosh \left(\lambda_{1}\right) \\
& +\lambda_{2}\left(\lambda_{1}^{2}-\Omega^{2} S\right)\left\{\lambda_{2}^{2}+\Omega^{2}\left(S+\frac{1}{\lambda^{2}}\right)\right\} \sin \left(\lambda_{2}\right) \\
& \left.\cdot \sinh \left(\lambda_{1}\right)+\lambda_{1}\left(\lambda_{1}^{2}-\Omega^{2} S\right)\left\{\lambda_{1}^{2}-\Omega^{2}\left(S+\frac{1}{\lambda^{2}}\right)\right\}\right] \\
& =0 .
\end{aligned}
$$

\section{FE Formulation of Damped and Shear-Flexible Beck Column}

A FE formulation of Beck's column is briefly presented based on the dimensionless energy expression of (3) in this section, and Rayleigh damping is discussed in relation to internal and external damping terms in the next section.

Figure 2 shows Beck's column subjected to a follower force $P$ and a lateral impulse force $F^{*}(t)$ at the tip end which is modeled using 20 two-node shear-flexible Hermitian beam elements. The element model considered here consists of two nodes, and each node has two degrees of freedom with element length $d$. The nondimensional vertical displacement $v^{*}$ and rotation $\beta^{*}$ of a typical point within the element can be related to the nodal displacements using the third-order Hermitian interpolation polynomial as follows:

$$
\begin{aligned}
T v^{*}(\xi) & =h_{11} v^{* p}+h_{12} \beta^{* p}+h_{13} v^{* q}+h_{14} \beta^{* q}, \\
T \beta^{*}(\xi) & =f_{11} v^{* p}+f_{12} \beta^{* p}+f_{13} v^{* p}+f_{14} \beta^{* q}, \\
v^{* p} & =v^{*}(0), \\
\beta^{* p} & =\beta^{*}(0), \\
v^{* q} & =v^{*}(d), \\
\beta^{* q} & =\beta^{*}(d), \\
h_{11} & =2 \xi^{3}-3 \xi^{2}-12 s \xi+T ; \\
h_{12} & =\left\{\xi^{3}-2(1+3 s) \xi^{2}+(1+6 s) \xi\right\} d ;
\end{aligned}
$$




$$
\begin{aligned}
h_{13} & =-2 \xi^{3}+3 \xi^{2}+12 s \xi \\
h_{14} & =\left\{\xi^{3}-(1-6 s) \xi^{2}-6 s \xi\right\} d \\
f_{11} & =\frac{\left(6 \xi^{2}-6 \xi\right)}{d} ; \\
f_{12} & =3 \xi^{2}-4(1+3 s) \xi+T \\
f_{13} & =-f_{11} \\
f_{14} & =3 \xi^{2}-2(1-6 s) \xi \\
T & =(1+12 s) \\
s & \equiv \frac{E I}{\left(G A_{s} d^{2}\right)} \\
\xi & =\frac{x}{d},
\end{aligned}
$$

where it is noted that the element length $d$ in the lowercase " $s$ " in (17) is calculated by dividing the dimensionless column length of 1.0 by the element number.

Now, substituting the above interpolation functions into (3) and integrating it, the equations of motion are expressed as

$$
\begin{aligned}
\mathbf{M U ̈}+\mathbf{C} \dot{U}+\left[\mathbf{K}_{\mathbf{E}}-P^{*} \mathbf{K}_{\mathbf{G}}+\alpha P^{*} \mathbf{K}_{\mathrm{NC}}\right] \mathbf{U} & =\mathbf{F}^{*}(\mathbf{t}), \\
\mathbf{M} & =\sum_{\mathbf{e}} \mathbf{m}_{\mathbf{e}}, \\
\mathbf{K}_{\mathbf{E}} & =\sum_{\mathbf{e}} \mathbf{k}_{\mathbf{e}}, \\
\mathbf{K}_{\mathbf{G}} & =\sum_{\mathbf{e}} \mathbf{k}_{\mathbf{g}}, \\
\mathbf{K}_{\mathrm{NC}} & =\sum_{\mathbf{e}} \mathbf{k}_{\mathbf{n c}},
\end{aligned}
$$

where the $4 \times 4$ element mass matrix $\mathbf{m}_{\mathbf{e}}$ and elastic and geometric stiffness matrices $\mathbf{k}_{\mathbf{e}}, \mathbf{k}_{\mathbf{g}}$ are

$$
\begin{aligned}
& \mathbf{m}_{\mathbf{e}}=\frac{d}{420 T^{2}}\left[\begin{array}{rrrr}
m_{1} & m_{2} & m_{3} & m_{4} \\
& m_{5} & -m_{4} & m_{6} \\
& m_{1} & -m_{2} \\
& & \\
\text { symm. } & & m_{5}
\end{array}\right] \\
& +\frac{1}{30 T^{2} \lambda^{2} d}\left[\begin{array}{rrrr}
r_{1} & r_{2} & -r_{1} & r_{2} \\
& r_{3} & -r_{2} & r_{4} \\
& & r_{1} & -r_{2} \\
& s y m m . & & r_{3}
\end{array}\right], \\
& \mathbf{k}_{\mathbf{g}}=\frac{1}{T^{2}}\left[\begin{array}{rrr}
a_{1} \\
a_{2} & -a_{1} & a_{2} \\
a_{3} & -a_{2} & -a_{4} \\
& a_{1} & -a_{2} \\
\text { symm. } & & a_{3}
\end{array}\right],
\end{aligned}
$$

$$
\mathbf{k}_{\mathbf{n c}}=\frac{1}{T}\left[\begin{array}{cccc}
\cdot & \cdot & \cdot & \cdot \\
\cdot & \cdot & \cdot & \cdot \\
-a_{5} & -a_{6} & a_{5} & 1+a_{6} \\
\cdot & \cdot & \cdot & \cdot
\end{array}\right]
$$

where

$$
\begin{aligned}
& m_{1}=12\left(13+294 s+1680 s^{2}\right) \\
& m_{2}=2\left(11+231 s+1260 s^{2}\right) \\
& m_{3}=18\left(3+84 s+560 s^{2}\right) \\
& m_{4}=-\left(13+378 s+2520 s^{2}\right) d ; \\
& m_{5}=4\left(1+21 s+126 s^{2}\right) d^{2} \\
& m_{6}=-3\left(1+28 s+168 s^{2}\right) d^{2} \\
& r_{1}=36 \\
& r_{2}=3(1-60 s) d ; \\
& r_{3}=4\left(1+15 s+360 s^{2}\right) d^{2} \\
& r_{4}=-\left(1+60 s-720 s^{2}\right) d^{2} \\
& a_{6}=6 s \\
& a_{5} \\
& a_{1}=\frac{6}{5 d}\left(1+20 s+120 s^{2}\right) \\
& a_{2}=\frac{1}{10} \\
& a_{3}
\end{aligned}
$$

The load correction stiffness matrix $\mathbf{k}_{\mathbf{n c}}$ is not null only for the element including the tip end, as shown in (19c), and the damping matrix is discussed in the next section. The matrix formulation presented is similar to that by Attard et al. [19] but was reproduced for the sake of completeness.

First, the critical divergence load $P_{d}^{*}$ is evaluated from the following equilibrium equations of Beck's column:

$$
\mathbf{K}_{\mathbf{E}} \mathbf{U}=P^{*}\left[\mathbf{K}_{\mathbf{G}}-\alpha \mathbf{K}_{\mathbf{N C}}\right] \mathbf{U} \text {. }
$$

In the case of the undamped system, by putting $\mathbf{U}=$ $e^{\Omega t^{*}} \mathbf{H}$, the equations of motion are written as

$$
\Omega^{2} \mathbf{M H}+\left[\mathbf{K}_{\mathbf{E}}-P^{*} \mathbf{K}_{\mathbf{G}}+\alpha P^{*} \mathbf{K}_{\mathbf{N C}}\right] \mathbf{H}=\mathbf{0} .
$$

As $P^{*}$ increases, stability is lost when two consecutive eigenvalues of $\Omega$ become equal at a finite critical value of 
TABLE 1: Material and geometric properties of the Beck's column model.

\begin{tabular}{|c|c|c|c|c|c|c|}
\hline Property & & Unit & & \multicolumn{3}{|c|}{ Dimensionless unit } \\
\hline Cross-sectional area, $A$ & & $1.2 \times 10^{-4} \mathrm{~m}^{2}$ & & \multicolumn{3}{|c|}{1.0} \\
\hline Moment of inertia, $I$ & \multicolumn{3}{|c|}{$1.0 \times 10^{-9} \mathrm{~m}^{4}$} & \multicolumn{3}{|c|}{1.0} \\
\hline Elastic modulus, $E$ & \multicolumn{3}{|c|}{$10.0 \mathrm{Gpa}$} & \multicolumn{3}{|c|}{1.0} \\
\hline Poisson's ratio, $v$ & \multicolumn{3}{|c|}{0.3} & \multicolumn{3}{|c|}{0.3} \\
\hline Mass density, $\rho$ & \multicolumn{3}{|c|}{$1.0 \times 10^{3} \mathrm{~kg} \mathrm{~m}^{-3}$} & \multicolumn{3}{|c|}{1.0} \\
\hline Total length of column, $l$ & $1.0 \mathrm{~m}$ & $71.4 \mathrm{~mm}$ & $35.7 \mathrm{~mm}$ & 1.0 & 1.0 & 1.0 \\
\hline Slenderness ratio, $\lambda$ & 346.4 & 24.74 & 12.37 & $\infty$ & 24.74 & 12.37 \\
\hline Shear parameter, $S$ & $2.55 \times 10^{-5}$ & 0.005 & 0.02 & 0.0 & 0.005 & 0.02 \\
\hline
\end{tabular}

TABLE 2: Dimensionless natural frequencies and damping coefficients of a cantilever beam.

\begin{tabular}{|c|c|c|c|c|}
\hline \multirow{2}{*}{ Property } & & \multicolumn{3}{|c|}{ Shear parameter, $S$} \\
\hline & & $0.0\left(2.55 \times 10^{-5}\right)$ & 0.005 & 0.02 \\
\hline \multirow{2}{*}{ Dimensionless natural frequency } & $\omega_{1}^{*}$ & $3.5160(3.5157)$ & 3.4632 & 3.3203 \\
\hline & $\omega_{2}^{*}$ & $22.035(22.022)$ & 20.004 & 16.210 \\
\hline \multirow{2}{*}{$\begin{array}{l}\text { Dimensionless damping coefficients corresponding to a proportional } \\
\text { damping ratio of } 0.1 \%\end{array}$} & $\gamma_{1}^{*}$ & $6.064 \times 10^{-3}$ & $5.904 \times 10^{-3}$ & $5.512 \times 10^{-3}$ \\
\hline & $\gamma_{2}^{*}$ & $7.828 \times 10^{-5}$ & $8.523 \times 10^{-5}$ & $1.024 \times 10^{-4}$ \\
\hline \multirow{2}{*}{$\begin{array}{l}\text { Dimensionless damping coefficients corresponding to a proportional } \\
\text { damping ratio of } 2 \%\end{array}$} & $\gamma_{1}^{*}$ & $1.213 \times 10^{-1}$ & $1.181 \times 10^{-1}$ & $1.102 \times 10^{-1}$ \\
\hline & $\gamma_{2}^{*}$ & $1.566 \times 10^{-3}$ & $1.705 \times 10^{-3}$ & $2.048 \times 10^{-3}$ \\
\hline
\end{tabular}

The values in parentheses denote the shear parameter and frequencies evaluated for the column given in Table 1.

$P^{*}$. Beyond this value, known as the undamped flutter load $P_{\mathrm{fl}}^{*}$, the perturbed motion of the system displays explosively diverging oscillations with increasing amplitudes, while the critical eigenvalues become complex conjugates.

Finally, the flutter load $P_{\text {fl_d }}^{*}$ of the damped flutter system is determined by adding the damping forces to (22) and solving the quadratic eigenproblem:

$$
\Omega^{2} \mathbf{M H}+\Omega \mathbf{C H}+\left[\mathbf{K}_{\mathbf{E}}-P^{*} \mathbf{K}_{\mathbf{G}}+\alpha P^{*} \mathbf{K}_{\mathbf{N C}}\right] \mathbf{H}=\mathbf{0} .
$$

\section{Estimation of Damping Coefficients for Stability Analysis of Beck's Columns}

In deriving (18), it should be noted that integrating the damping-related terms in (3) leads to

$$
\begin{aligned}
& \int_{o}^{d}\left\{\gamma_{1}^{*}\left(\dot{v}^{*} \delta v^{*}+\frac{\dot{\beta}^{*} \delta \beta^{*}}{\lambda^{2}}\right)+\gamma_{2}^{*}\left(\dot{\beta}^{* \prime} \delta \beta^{* \prime}\right.\right. \\
& \left.\left.+\frac{\left(\dot{v}^{* \prime}-\dot{\beta}^{*}\right) \delta\left(v^{* \prime}-\beta^{*}\right)}{S}\right)\right\} d x^{*} \\
& =\delta \mathbf{u}_{\mathbf{e}}\left(\gamma_{1}^{*} \mathbf{m}_{\mathbf{e}}+\gamma_{2}^{*} \mathbf{k}_{\mathbf{e}}\right) \dot{\mathbf{u}}_{\mathbf{e}}
\end{aligned}
$$

which means that the damping matrix in (9) due to external and internal damping terms can be expressed in the form of Rayleigh damping as follows:

$$
\mathbf{C}=\gamma_{1}^{*} \mathbf{M}+\gamma_{2}^{*} \mathbf{K}_{\mathbf{E}}
$$

In other words, the external and internal damping coefficients $\gamma_{1}^{*}, \gamma_{2}^{*}$ are directly connected to Rayleigh damping coefficients. Hence, the damping coefficients can be easily determined, under the assumption of being proportional damping, as follows:

$$
\begin{aligned}
& 2 \xi \omega_{1}^{*}=\gamma_{1}^{*}+\left(\omega_{1}^{*}\right)^{2} \gamma_{2}^{*}, \\
& 2 \xi \omega_{2}^{*}=\gamma_{1}^{*}+\left(\omega_{2}^{*}\right)^{2} \gamma_{2}^{*},
\end{aligned}
$$

where $\omega_{1}^{*}, \omega_{2}^{*}$ denote the first and second dimensionless natural frequencies of a cantilever beam, respectively, and $\gamma_{1}^{*}, \gamma_{2}^{*}$ can be obtained by solving simultaneous equation (26). It is worth noting from (26) that $\gamma_{1}^{*}, \gamma_{2}^{*}$ under the assumption of Rayleigh damping increase in direct proportion to the damping ratio $\xi$. Additionally, in the case of isotropic materials with a rectangular section, the following relationship between the slenderness ratio $\lambda$ and the shear parameter $S$ can be obtained:

$$
S=\frac{E /\left(G f_{s}\right)}{\lambda^{2}}=2(1+\nu) \cdot \frac{12+11 \nu}{10(1+\nu) \lambda^{2}}=\frac{3.06}{\lambda^{2}},
$$

where $f_{s}=10(1+v) /(12+11 \nu), v=0.3$

Table 1 presents the material and geometric properties of Beck's column model and the corresponding dimensionless data, and Table 2 lists dimensionless natural frequencies and damping coefficients for $S$ chosen to be $0.0,0.005$, and 0.02 in this study. For a column length of $1.0 \mathrm{~m}$, the shear parameter $S$ and the slenderness ratio $\lambda$ of the cantilever column model are evaluated as $2.55 \times 10^{-5}$ and 346.4, respectively, in which $S$ is so small that it is expected that the shear effect can be neglected. Table 2 shows that the two natural frequencies calculated for $S=0.0,2.55 \times 10^{-5}$ are almost identical, which means that $S$ and $\lambda$ can be regarded as infinity and zero, respectively. 
TABLE 3: Damped flutter loads of shear-rigid Beck's columns with variation of two damping coefficients for $\alpha=1.0$.

\begin{tabular}{|c|c|c|c|c|c|c|c|}
\hline$\gamma_{2}^{*}$ & 0.0 & 0.001 & 0.01 & $\begin{array}{l}\gamma_{1}^{*} \\
0.1\end{array}$ & 1.0 & 10.0 & 100.0 \\
\hline 0.0 & $20.05(16.05)$ & $20.05(16.05)$ & $20.05(16.05)$ & $20.05(16.05)$ & $20.11(16.08)$ & $24.27(17.63)$ & $37.21(19.99)$ \\
\hline 0.0001 & $10.94(9.870)$ & $12.88^{\ddagger}(11.33)$ & $17.55^{\dagger}(14.53)$ & $19.91(15.97)$ & $20.11(16.08)$ & $24.28(17.63)$ & 37.21 (19.99) \\
\hline 0.001 & $10.94(9.870)$ & $11.19(10.06)$ & $12.88^{\ddagger}(11.33)$ & $17.56^{\dagger}(14.53)$ & 19.98 (15.99) & 24.38 (17.63) & 37.21 (19.98) \\
\hline 0.01 & $10.97(9.870)$ & $10.99(9.890)$ & $11.22(10.07)$ & $12.93^{\ddagger}(11.33)$ & $17.80^{\dagger}(14.58)$ & $25.20(17.60)$ & $37.21(19.94)$ \\
\hline 0.1 & $13.64(9.870)$ & $13.64(9.872)$ & $13.68(9.890)$ & $14.08(10.07)$ & $17.33(11.40)$ & 32.10 (15.98) & 37.38 (19.48) \\
\hline 0.2 & $21.51(9.870)$ & $21.51(9.871)$ & $21.56(9.880)$ & 21.98 (9.969) & $25.86(10.76)$ & $41.31(14.65)$ & 37.85 (19.02) \\
\hline
\end{tabular}

Values in round brackets denote damped flutter loads in the case of $\alpha=0.5$.

To evaluate shear and rotary inertia effects on divergence and flutter loads, the three slenderness ratios corresponding to three shear parameters are determined using (27) and displayed in Table 1 . In addition, the dimensionless natural frequencies and damping coefficients corresponding to $\xi=$ $0.001,0.02$ are calculated from (15) and (26), and the results are presented in Table 2. Conclusively, after determining dimensionless damping coefficients, the dimensionless flutter and divergence loads of Beck's column model can be determined from the closed-form solutions of (9) and (13) and from the FE solutions of (23) and (21).

To explore the external and internal damping effects on the flutter behaviors of Beck's columns effectively, three representative cases of external and internal damping coefficients, namely, the extreme case of external damping only $\left(\gamma_{1}^{*}\right.$ in the case of $2 \%$ proportional damping, $\gamma_{2}^{*}=0$ ), the extreme case of small internal damping only $\left(\gamma_{1}^{*}=0, \gamma_{2}^{*}\right.$ in the case of $0.1 \%$ proportional damping), and the practical case of external and internal damping $\left(\gamma_{1}^{*}, \gamma_{2}^{*}\right.$ in the case of $2 \%$ proportional damping), have been selected based on Table 2, as follows:

Case 1 of external damping only:

$$
\begin{aligned}
& \gamma_{1}^{*}=1.213 \times 10^{-1}, \gamma_{2}^{*}=0 \text { for } S=0.0, \\
& \gamma_{1}^{*}=1.181 \times 10^{-1}, \gamma_{2}^{*}=0 \text { for } S=0.005, \\
& \gamma_{1}^{*}=1.102 \times 10^{-1}, \gamma_{2}^{*}=0 \text { for } S=0.02 .
\end{aligned}
$$

Case 2 of internal damping only:

$$
\begin{aligned}
& \gamma_{1}^{*}=0, \gamma_{2}^{*}=7.828 \times 10^{-5} \text { for } S=0.0, \\
& \gamma_{1}^{*}=0, \gamma_{2}^{*}=8.523 \times 10^{-5} \text { for } S=0.005, \\
& \gamma_{1}^{*}=0, \gamma_{2}^{*}=1.024 \times 10^{-4} \text { for } S=0.02 .
\end{aligned}
$$

Case 3 of external and internal damping:

$$
\begin{aligned}
& \gamma_{1}^{*}=1.213 \times 10^{-1}, \gamma_{2}^{*}=1.566 \times 10^{-3} \text { for } S=0.0, \\
& \gamma_{1}^{*}=1.181 \times 10^{-1}, \gamma_{2}^{*}=1.705 \times 10^{-3} \text { for } S= \\
& 0.005, \\
& \gamma_{1}^{*}=1.102 \times 10^{-1}, \gamma_{2}^{*}=2.048 \times 10^{-3} \text { for } S=0.02 .
\end{aligned}
$$

In this, Cases 1,2 , and 3 have been chosen to investigate the effects of external damping only, very small internal damping only, and realistic damping containing both external and internal damping forces, respectively. Here, the damped flutter loads of Beck's columns corresponding to Cases 1, 2, and 3 are denoted by $P_{\mathrm{fl} \_d 1}^{*}$ for $\xi=0.02, P_{\mathrm{fl} \_d 2}^{*}$ for $\xi=0.001$, and $P_{\mathrm{fl} \_d 3}^{*}$ for $\xi=0.02$, respectively.

In the remaining part of this section, a parametric study is performed to demonstrate that the three damping cases can indeed be regarded as representative values of internal and external damping.

Table 3 shows how the damped flutter loads $P_{\mathrm{fl} d}^{*}$ of shearrigid Beck's columns vary for $\alpha=1.0$ as the two damping coefficients increase independently, where values in round brackets denote the damped flutter loads in the case of $\alpha=$ 0.5 . From Table 2, it is observed that the damped flutter load tends to decease with introduction of internal damping, but external damping alleviates this tendency, which has been well known. In particular, it should be noticed that flutter loads hardly alter in the practical range when the ratio of two damping coefficients $\gamma_{1}^{*} / \gamma_{2}^{*}$ is maintained constant as 10.0 and 100.0 (marked by the superscripts ${ }^{\ddagger}$ and ${ }^{\dagger}$, resp.).

To investigate this tendency further, Table 4 shows fluctuations of $P_{\text {fl } d}^{*}$ for $S=0.0,0.02$ with increasing damping ratio $\xi$, where values in round brackets denote the flutter loads for $\alpha=0.5$. It should be noted that the damped flutter loads in the second, third, and fourth columns of Table 4 are evaluated based on the three damping conditions of taking $\gamma_{1}^{*}$ with $\gamma_{2}^{*}$ being set as zero, $\gamma_{2}^{*}$ with $\gamma_{1}^{*}$ being set as zero, and both $\gamma_{1}^{*}$ and $\gamma_{2}^{*}$ from two coefficients $\gamma_{1}^{*}, \gamma_{2}^{*}$ calculated for $\xi$ ranging from 0.01 to $100 \%$. Also note that the three conditions correspond to the $\gamma_{1}^{*} / \gamma_{2}^{*}$ ratio being constant at $\infty, 0.0$, and 77.5.

Interestingly, Table 4 demonstrates that overall the damped flutter loads are hardly sensitive to the proportional damping ratio $\xi$ but strongly depend on the damping ratio, $\gamma_{1}^{*} / \gamma_{2}^{*}$, except for the last low corresponding to $100 \%$ damping ratio. That is, it is observed from the second column that the flutter loads $P_{\mathrm{fl} \_d 1}^{*}$ with consideration of external damping only remain around the undamped flutter load $P_{\mathrm{fl}}^{*}, 20.05$ (16.05) and 12.12 (10.95) for $S=0.0,0.02$ despite the increased external damping. Also, the flutter loads $P_{\mathrm{fl} \_d 2}^{*}$ of the third column with increasing internal damping only are nearly equal to the damped flutter load, 10.94 (9.870) and 8.083 (7.529) under the extreme condition of very small internal damping only. In particular, the fourth column shows that the damped flutter loads $P_{\mathrm{fl} \_d 3}^{*}$ under both damping coefficients are around $17.03(14.20)$ and 10.90 (9.969) at a damping ratio $\xi$ of up to $10 \%$. Here it should be mentioned that Bolotin and Zhinzher [20] and Herrmann and Jong [21] noticed the importance of the ratio between internal and 
TABLE 4: Damped flutter loads of Beck's columns for $S=0.0,0.02$ with increase in the damping ratio for $\alpha=1.0$.

\begin{tabular}{lcccccc}
\hline Damping ratio (\%) & \multicolumn{2}{c}{ External damping only, $P_{\mathrm{fl} d 1}^{*}$} & \multicolumn{2}{c}{ Internal damping only, $P_{\mathrm{fl} d 2}^{*}$} & \multicolumn{3}{c}{ External and internal damping, $P_{\text {fl_d3 }}^{*}$} \\
$\xi$ & $S=0.0$ & $S=0.02$ & $S=0.0$ & $S=0.02$ & $S=0.0$ & $S=0.02$ \\
\hline 0.01 & $20.05(16.05)$ & $12.12(10.95)$ & $10.94(9.870)$ & $8.083(7.529)$ & $17.03(14.20)$ & $10.90(9.967)$ \\
0.1 & $20.05(16.05)$ & $12.12(10.95)$ & $10.94(9.870)$ & $8.083(7.529)$ & $17.03(14.20)$ & $10.90(9.967)$ \\
2.0 & $20.05(16.05)$ & $12.12(10.95)$ & $10.94(9.870)$ & $8.084(7.529)$ & $17.03(14.20)$ & $10.90(9.969)$ \\
5.0 & $20.05(16.06)$ & $12.13(10.95)$ & $10.95(9.870)$ & $8.088(7.530)$ & $17.06(14.20)$ & $10.92(9.974)$ \\
8.0 & $20.06(16.06)$ & $12.13(10.95)$ & $10.95(9.870)$ & $8.096(7.531)$ & $17.10(14.21)$ & $10.97(9.985)$ \\
10.0 & $20.07(16.06)$ & $12.14(10.96)$ & $10.96(9.870)$ & $8.103(7.532)$ & $17.15(14.21)$ & $11.01(9.994)$ \\
100.0 & $21.83(16.80)$ & $13.78(11.72)$ & $12.6(9.870)$ & $10.04(7.716)$ & $26.22(15.13)$ & $18.30(11.05)$ \\
\hline
\end{tabular}

Values in round brackets denote the damped flutter loads in the case of $\alpha=0.5$.

external damping, and, recently, Kirillov and Seyranian [22] demonstrated this dependence of damped flutter loads on the damping ratio in an analytical form. From these observations, the following summary can be made:

(1) Two damping coefficients $\gamma_{1}^{*}, \gamma_{2}^{*}$ increase in direct proportion to the damping ratio $\xi$ under the assumption of Rayleigh damping.

(2) The damped flutter loads are closely dependent on the $\gamma_{1}^{*} / \gamma_{2}^{*}$ ratio.

(3) The damped flutter loads hardly vary when two coefficients $\gamma_{1}^{*}, \gamma_{2}^{*}$ are increased in the practical range of $\gamma_{1}^{*} / \gamma_{2}^{*}$ ratio maintained at a specific value.

(4) Cases 1 and 2 represent two extreme cases of external and internal damping only and the flutter loads of shear-rigid Beck's columns for the two cases will be similar to 20.05 (16.05) and 10.94 (9.870), respectively, despite the increase in the corresponding damping coefficients.

(5) Particularly, Case 3, which corresponds to a $2 \%$ damping ratio under Rayleigh damping, represents the most realistic damping case and the damped flutter loads for Case 3 are unchanged 17.03, (14.20) with increasing damping ratio $\xi$.

\section{Stability Maps of Damped and Shear-Flexible Beck's Columns}

Stability maps of damped and shear-flexible Beck's columns subjected to a subtangential follower force are presented and compared in this section. Stability maps of subtangentially loaded and damped Beck's column for $S=0.0,0.005$, and 0.02 have been constructed from analytical solutions using Mathematica [30] as the subtangentiality $\alpha$ is varied in the range of $0.0 \leq \alpha \leq 1.0 . \alpha$ is divided into five ranges according to the instability characteristics (Table 5), and the resulting stability maps are presented in Figures 3-6. Additionally, Table 6 lists the divergence and flutter loads of Beck's column at increments and important transition points of $\alpha$ related to Figures 3-6. The figures and Table 6 indicate that not only the critical divergence loads but also the undamped and damped flutter loads are greatly reduced as the shear parameter $S$ is increased.
TABLE 5: Ranges of $\alpha$ according to instability characteristics.

\begin{tabular}{lccc}
\hline & $S=0.0$ & $S=0.005$ & $S=0.02$ \\
\hline Range 1 & $0.0<\alpha \leq 0.321$ & $0.0<\alpha \leq 0.318$ & $0.0<\alpha \leq 0.306$ \\
Range 2 & $0.321<\alpha \leq 0.354$ & $0.318<\alpha \leq 0.352$ & $0.306<\alpha \leq 0.345$ \\
Range 3 & $0.354<\alpha \leq 0.439$ & $0.352<\alpha \leq 0.433$ & $0.345<\alpha \leq 0.414$ \\
Range 4 & $0.439<\alpha \leq 0.5$ & $0.433<\alpha \leq 0.488$ & $0.414<\alpha \leq 0.456$ \\
Range 5 & $0.5<\alpha \leq 1.0$ & $0.488<\alpha \leq 1.0$ & $0.456<\alpha \leq 1.0$ \\
\hline
\end{tabular}

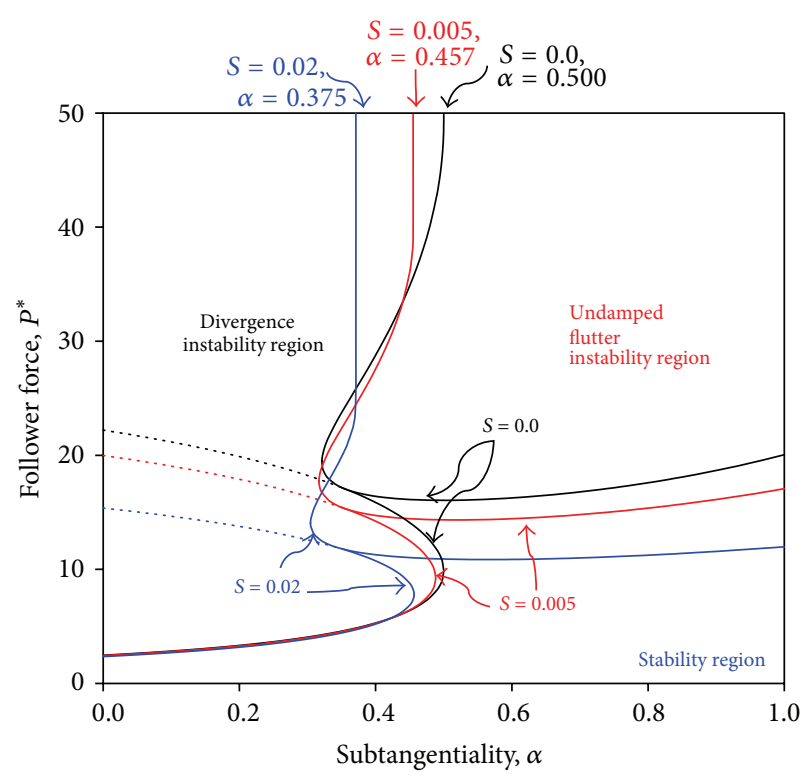

FIGURE 3: Stability regions of shear deformable Beck's column with variation of $\alpha$.

Firstly, Figure 3 shows that an undamped Beck's column with $S=0.0,0.005$, and 0.02 may be stable or unstable depending on the range of $\alpha$ as follows:

(1) Range 1: it loses its stability by divergence at load levels higher than $P_{d 1}^{*}$.

(2) Range 2: it is first stable with increasing follower force but becomes unstable due to divergence at $P_{d 1}^{*}$, then due to dynamic flutter at $P_{\mathrm{fl}}^{*}$, and again due to divergence at load levels higher than $P_{\mathrm{fl} 2}^{*}$. 


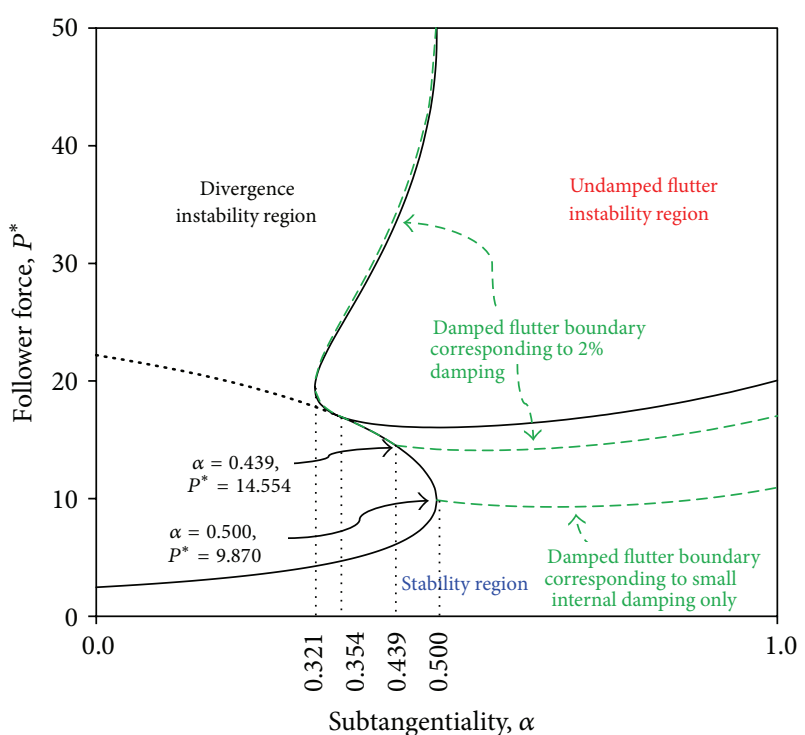

FIGURE 4: Stability regions of shear-rigid Beck's column with variation of $\alpha$.

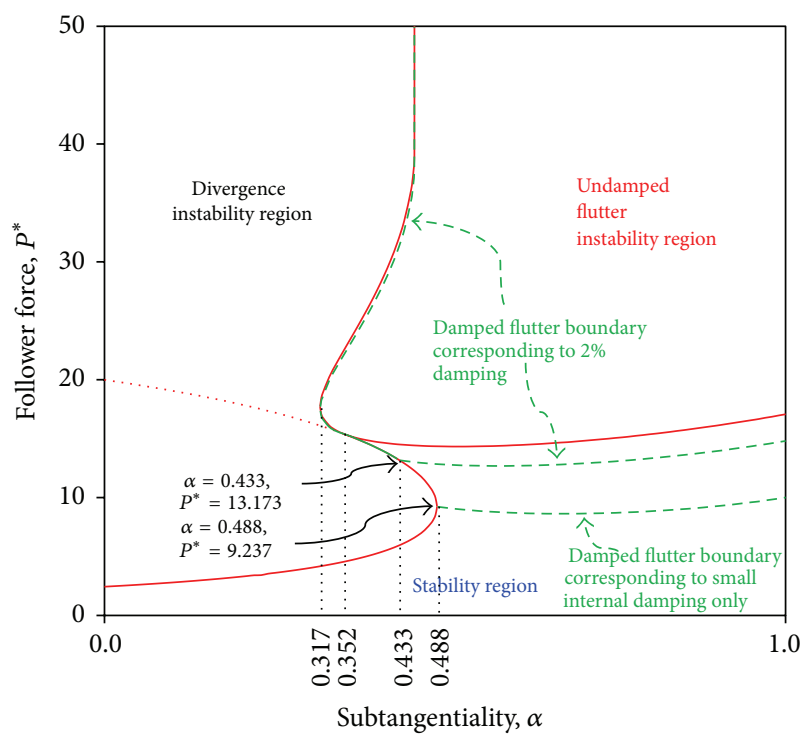

Figure 5: Stability regions of Beck's column for $S=0.005$ with variation of $\alpha$.

(3) Ranges 3 to 4 : it is stable initially and then loses its stability due to divergence at $P_{d 1}^{*}$ but restores its stability at $P_{d 2}^{*}$ again (restabilization) and finally becomes unstable due to flutter at $P_{\mathrm{fl} 1}^{*}$.

(4) Range 5: it loses its stability at $P_{\text {fl1 }}^{*}$.

Three stability maps for damped Beck's columns with $S=$ $0.0,0.005$, and 0.02 are displayed in Figures 4-6, in which three damping cases are taken into account. The stability map for Case 1 of external damping only is almost identical to that for the undamped case, and so no additional description is necessary. For Case 2 (small internal damping only), its

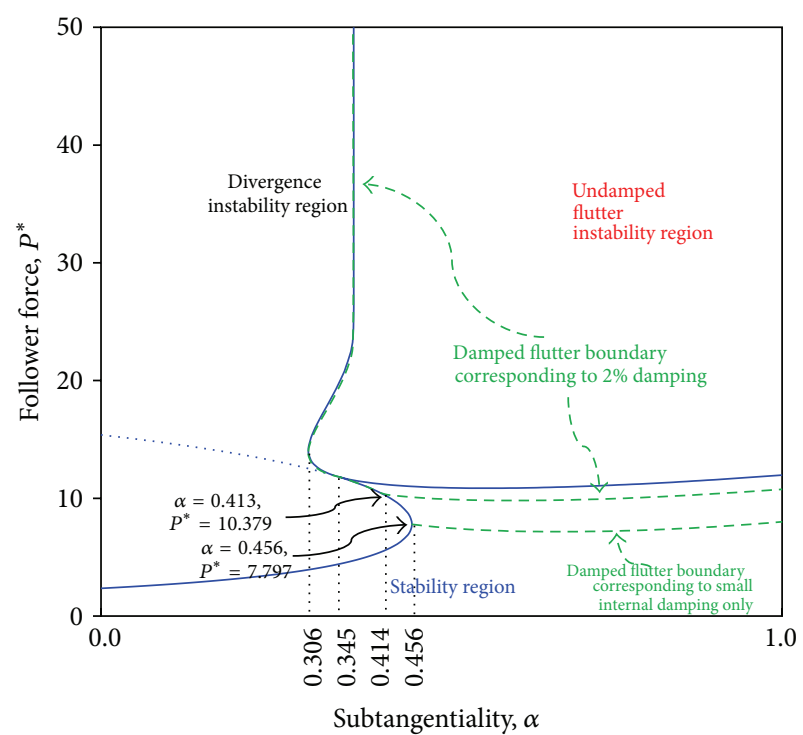

FIGURE 6: Stability regions of Beck's column for $S=0.02$ with variation of $\alpha$.

instability behaviors can be addressed depending on $\alpha$ as follows:

(1) Ranges 1 and 2: it is the same as those of the undamped system.

(2) Ranges 3 and 4: it is stable initially, then loses its stability due to divergence at $P_{d 1}^{*}$, and becomes unstable due to quiet flutter at $P_{d 2}^{*}$ and then due to violent flutter at $P_{\mathrm{fl} 1}^{*}$. Finally it is again governed by divergence at much higher values of $P_{\mathrm{fl} 2}^{*}$.

(3) Range 5: it loses its stability due to quiet flutter at a damped flutter load $P_{\mathrm{fl} \_d 2}^{*}$ and due to violent flutter at $P_{\mathrm{fl} 1}^{*}$.

The instability behaviors for Case 3 (external and internal damping) are as follows:

(1) Ranges 1 and 2: it is the same as those of the undamped system.

(2) Range 3: overall it is the same as those of Range 3 of Case 2.

(3) Range 4: it is the same as Range 3 until the follower force reaches $P_{d 2}^{*}$ but shows restabilization between $P_{d 2}^{*}$ and the damped flutter load $P_{\mathrm{fl} d 3}^{*}$ and quiet flutter instability between $P_{\mathrm{fl} \_d 3}^{*}$ and $P_{\mathrm{fl} 1}^{*}$.

(4) Range 5: it loses its stability due to quiet flutter at $P_{\text {fl_d }}^{*}$ and to violent flutter at $P_{\mathrm{fl} 1}^{*}$.

Table 7 shows the analytical solutions with FE solutions using 10, 20, and 40 elements for critical loads of shearflexible Beck's columns for $\alpha=0.4$ and 1.0. Clearly, FE solutions for shear deformable Beck's columns converge to the analytical solutions as the total number of elements is increased.

Finally, it is meaningful to investigate how not only the dimensionless frequencies $\Omega\left(\equiv \eta^{*}+i \omega^{*}\right)$ but also dynamic 


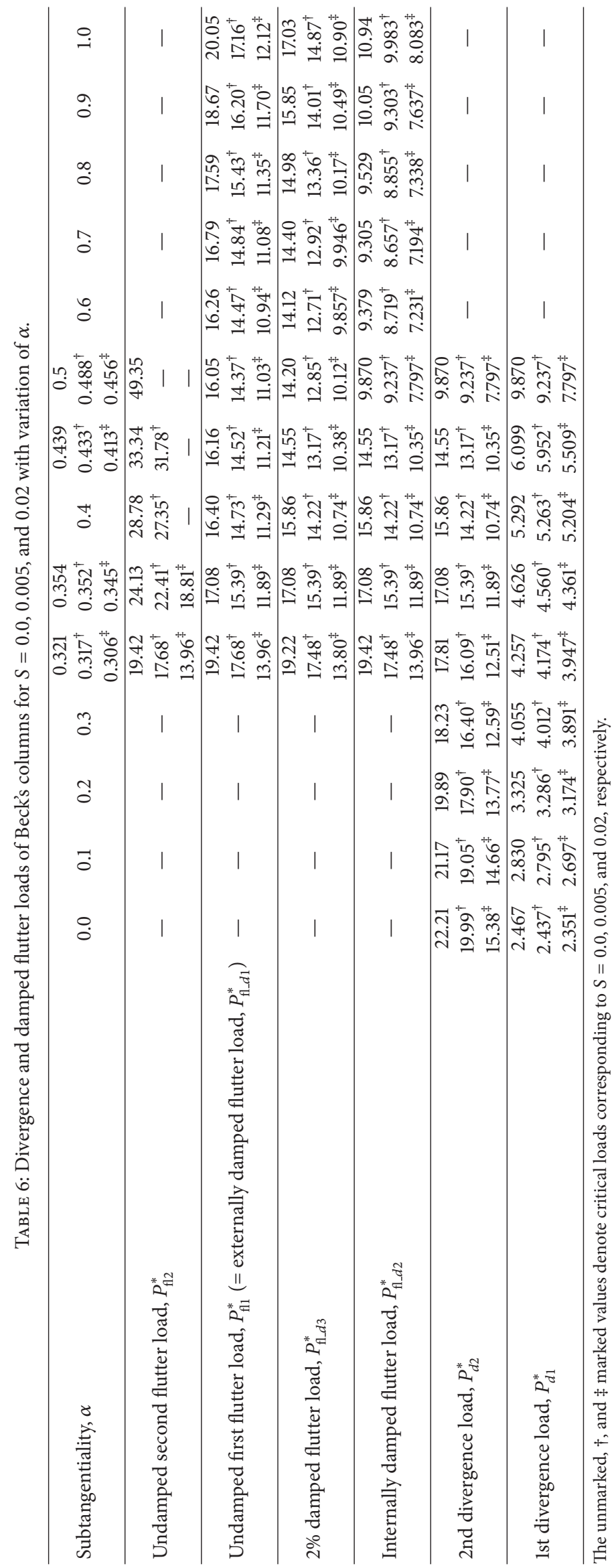


TABLE 7: Flutter and divergence loads of shear-flexible Beck's columns by analytical and FE solutions for $\alpha=0.4,1.0$.

\begin{tabular}{|c|c|c|c|c|c|c|}
\hline & & $\alpha=0.4$ & & & $\alpha=1.0$ & \\
\hline & $S=0.0$ & $S=0.005$ & $S=0.02$ & $S=0.0$ & $S=0.005$ & $S=0.02$ \\
\hline \multirow{4}{*}{$P_{\mathrm{fl} 1}^{*}\left(=P_{\mathrm{fl} \_d 1}^{*}\right)$} & 16.40 (exact) & 14.73 (exact) & 11.29 (exact) & 20.05 (exact) & 17.16 (exact) & 12.12 (exact) \\
\hline & $16.40(10)$ & $14.75(10)$ & $11.32(10)$ & $20.05(10)$ & $17.30(10)$ & $12.33(10)$ \\
\hline & $16.40(20)$ & $14.74(20)$ & $11.30(20)$ & $20.05(20)$ & $17.22(20)$ & $12.21(20)$ \\
\hline & $16.40(40)$ & $14.73(40)$ & $11.29(40)$ & $20.05(40)$ & $17.19(40)$ & $12.17(40)$ \\
\hline \multirow{4}{*}{$P_{\mathrm{fl} \_d 3}^{*}$} & 15.86 (exact) & 14.22 (exact) & 10.74 (exact) & 17.03 (exact) & 14.87 (exact) & 10.90 (exact) \\
\hline & $15.86(10)$ & $14.24(10)$ & $10.79(10)$ & $17.03(10)$ & $14.95(10)$ & $11.03(10)$ \\
\hline & $15.86(20)$ & $14.22(20)$ & $10.75(20)$ & $17.03(20)$ & $14.91(20)$ & $10.96(20)$ \\
\hline & $15.86(40)$ & $14.22(40)$ & $10.74(40)$ & $17.03(40)$ & $14.89(40)$ & $10.93(40)$ \\
\hline \multirow{4}{*}{$P_{\mathrm{fl} L d 2}^{*}$} & 15.86 (exact) & 14.22 (exact) & 10.74 (exact) & 10.94 (exact) & 10.04 (exact) & 8.083 (exact) \\
\hline & $15.86(10)$ & $14.24(10)$ & $10.79(10)$ & $10.94(10)$ & $10.05(10)$ & $8.117(10)$ \\
\hline & $15.86(20)$ & $14.22(20)$ & $10.75(20)$ & $10.94(20)$ & $10.04(20)$ & $8.100(20)$ \\
\hline & $15.86(40)$ & $14.22(40)$ & $10.74(40)$ & $10.94(40)$ & $10.04(40)$ & $8.091(40)$ \\
\hline \multirow{4}{*}{$P_{d 1}^{*}$} & 5.292 (exact) & 5.263 (exact) & 5.204 (exact) & \multirow{4}{*}{ - } & \multirow{4}{*}{ - } & \multirow{4}{*}{ - } \\
\hline & $5.292(10)$ & $5.263(10)$ & $5.201(10)$ & & & \\
\hline & $5.292(20)$ & $5.263(20)$ & $5.203(20)$ & & & \\
\hline & $5.292(40)$ & $5.263(40)$ & $5.203(40)$ & & & \\
\hline
\end{tabular}

Numbers in the parentheses are the numbers of elements used.

TABLE 8: Dimensionless frequencies of Beck's columns around damped flutter loads with an increase of $\xi$ for $\alpha=1.0$.

\begin{tabular}{|c|c|c|c|c|}
\hline \multirow{2}{*}{\multicolumn{2}{|c|}{$\underset{\xi}{\text { Damping ratio }}(\%)$}} & \multicolumn{3}{|c|}{ Dimensionless frequency, $\Omega$} \\
\hline & & Internal damping only, & External and internal damping, & External damping only, \\
\hline \multirow{2}{*}{0.01} & $0.99 P_{\text {fl_ } d}^{*}$ & $-2.03 \times 10^{-6}+5.36 i$ & $-1.72 \times 10^{-5}+7.46 \mathrm{i}$ & $-3.03 \times 10^{-4}+9.95 i$ \\
\hline & $1.01 P_{\mathrm{fl} \_d}^{*}$ & $9.21 \times 10^{-7}+5.41 i^{* *}$ & $2.65 \times 10^{-5}+7.61 i^{* *}$ & $0.84+11.00 i$ \\
\hline \multirow{2}{*}{2.0} & $0.99 P_{\mathrm{fl} \_d}^{*}$ & $-4.08 \times 10^{-4}+5.36 i$ & $-3.55 \times 10^{-3}+7.45 i$ & $-0.06+9.95 i$ \\
\hline & $1.01 P_{\mathrm{fl} \_d}^{*}$ & $1.82 \times 10^{-4}+5.41 i$ & $5.13 \times 10^{-3}+7.61 i$ & $0.78+11.00 i$ \\
\hline \multirow{2}{*}{8.0} & $0.99 P_{\mathrm{fl} \_d}^{*}$ & $-1.74 \times 10^{-3}+5.36 i$ & $-2.06 \times 10^{-2}+7.42 i$ & $-0.24+9.94 i$ \\
\hline & $1.01 P_{f l d}^{*}$ & $6.05 \times 10^{-4}+5.41 i$ & $1.14 \times 10^{-2}+7.56 i$ & $0.60+11.00 i$ \\
\hline
\end{tabular}

flutter behaviors vary using a time history analysis by the FE procedure with increasing internal and external damping coefficients.

Table 8 shows the dimensionless frequencies of shearrigid Beck's columns subjected to follower forces $1 \%$ more or less than the damped flutter loads, which are $P_{\mathrm{fl} d 1}^{*}(=20.05)$, $P_{\mathrm{fl} \_d 2}^{*}(=10.94)$, and $P_{\mathrm{fl} \_d 3}^{*}(=17.03)$ corresponding to three cases of external damping only, internal damping only, and external and internal damping for $\xi=0.001,0.02$, and 0.08 . Also, for time history analysis of the system, Beck's column model is discretized using 20 elements and subjected to a follower force and a small lateral load at the tip end is taken into account (see Figure 2), and a time history analysis is performed. Data of the beam model are shown in Table 1 and the time increment $\Delta t\left(\Delta t^{*}\right)$ is fixed to be $0.0001 \mathrm{~s}(0.000913)$. Three follower forces, $1.01 P_{\mathrm{fl} d 2}^{*}$ under internal damping only, $1.01 P_{\mathrm{fl} \_d 3}^{*}$ under external and internal damping, and $1.01 P_{\mathrm{fl} \_d 1}^{*}$ under external damping only for $\xi=0.001,0.02$, and 0.08 , act with a constant magnitude and the small disturbance $F\left(F^{*}\right)$ of $0.01 \mathrm{~N}(0.001)$ vanishes after $0.0001 \mathrm{~s}$ elapses, as shown in
Figure 7. Figures $8(a)-8(c)$ show the results of time history analyses of damped Beck's columns under a lateral impulse force and three follower forces.

It is apparent from the sign of the real part of frequencies in Table 8 that the system is stable under a follower force less than the damped flutter load but unstable under a follower force more than the damped load. In addition, it is noted from Table 8 and Figure 8 that internal damping makes the nonconservative system destabilized while external damping has a stabilizing effect on the system. Particularly, it is found that the positive real parts of the frequencies marked as $* *$ in Table 8 become very small in the case of a $0.01 \%$ damping ratio. Related to them, the results of dynamic analyses in Figures 8(a) and 8(b) suggest flutter behaviors with very slowly increasing amplitudes, which is why these dynamic phenomena are called quiet flutter.

Lastly, three damped Beck columns subjected to a lateral impulse force are analyzed under the same conditions as in Figure 8, except that the shear parameter $S$ is increased and the damping ratio is fixed at $2 \%$. The results of time 


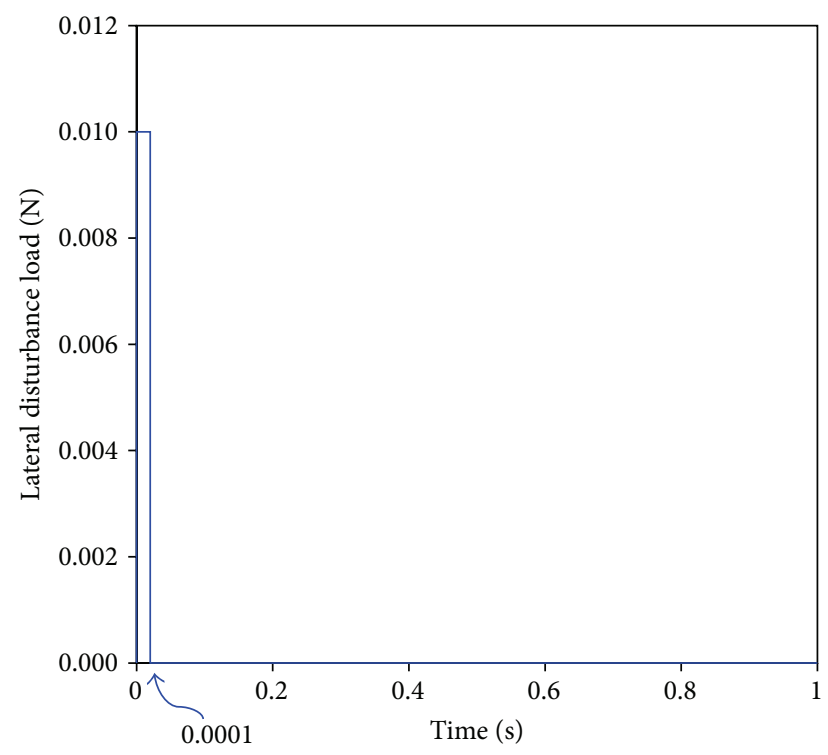

FIGURE 7: Lateral impulse force, $F(t)$.

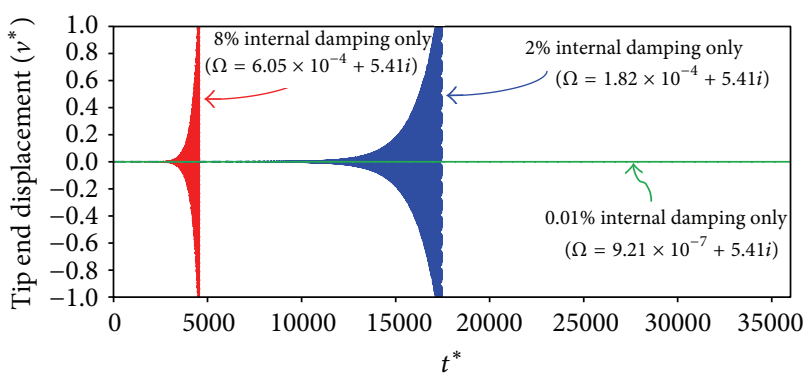

(a) $1.01 P_{\mathrm{fl} d 2}^{*}$ under internal damping only

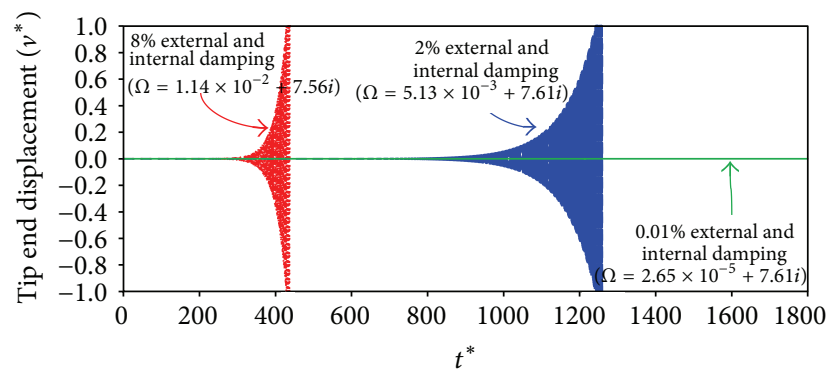

(b) $1.01 P_{\mathrm{fl} \_d 3}^{*}$ under external and internal damping

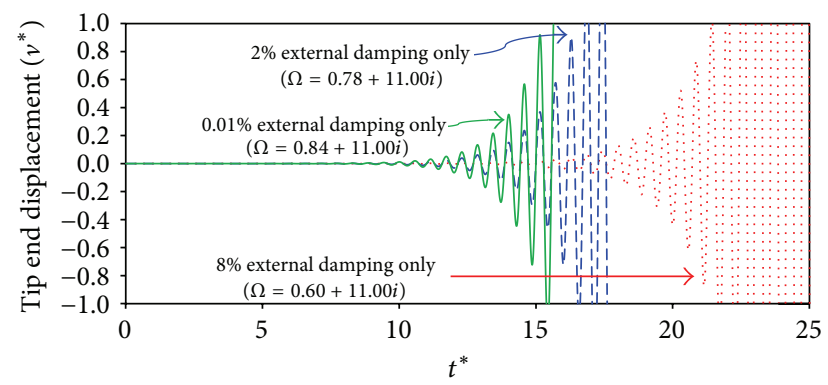

(c) $1.01 P_{\mathrm{fl} d 1}^{*}$ under external damping only

FIGURE 8: Results of time history analyses of shear-rigid and damped Beck's columns under a lateral impulse force.

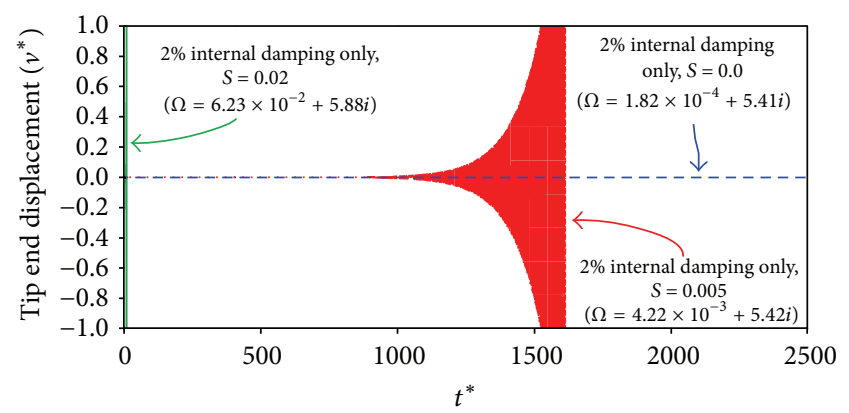

(a) $1.01 P_{\mathrm{fl} \_d 2}^{*}$ under $2 \%$ internal damping only with an increase in $S$

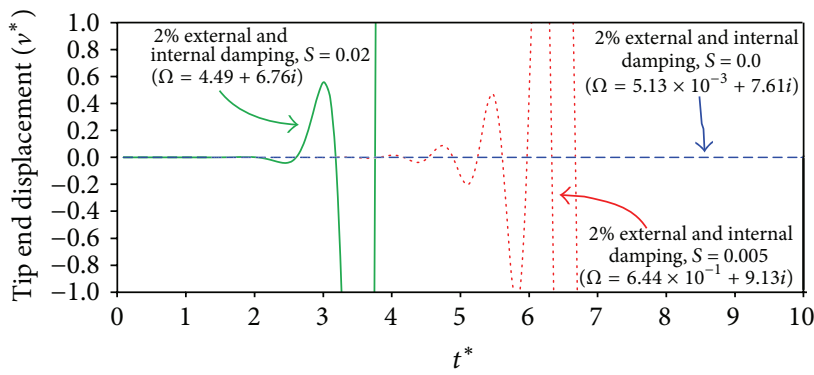

(b) $1.01 P_{\mathrm{fl} \_d 3}^{*}$ under $2 \%$ external and internal damping with an increase in

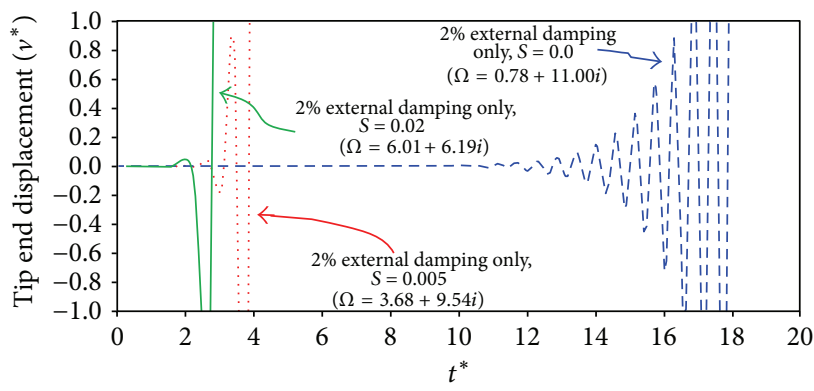

(c) $1.01 P_{\mathrm{fl} d}^{*} d 1$ under $2 \%$ external damping only with an increase in $S$

FIGURE 9: Results of time history analyses of shear-flexible and damped Beck's columns under a lateral impulse force.

history analyses at the tip end and the dimensionless complex frequencies are displayed in Figure 9. Figure 9 indicates that the real parts of the frequencies become large rapidly with increasing $S$, which results in early flutter instability of shearflexible Beck's columns.

\section{Conclusions}

A dimensionless frequency equation of shear-flexible and damped Beck's columns under a subtangential follow force was analytically derived in a closed form. Parametric studies were performed by varying two damping coefficients under the assumption of Rayleigh damping. It was demonstrated that three typical damping cases can be selected in association with flutter loads of Beck's columns. Finally, stability maps of shear-flexible and damped Beck's columns were constructed for the three damping cases and discussed in the practical range of damping coefficients and shear parameters. In addition, time history analysis was performed using 
a dimensionless FE procedure. The following conclusions can be drawn from this study:

(1) The dimensionless natural frequencies of a shear deformable beam can be calculated by fixing the shear parameter $S$ only, where the slenderness ratio $\lambda$ can be determined from a relation similar to (27).

(2) To construct a dimensionless stability map of shearflexible and damped Beck's columns, external and internal damping can be estimated from Case 3 without introduction of additional parameters.

(3) Under the assumption of Rayleigh damping, structural damping (Case 3 ) corresponding to $2 \%$ damping ratio provides the most realistic flutter loads of Beck columns, which make a big difference from flutter loads under external damping only (Case 1) and internal damping only (Case 2).

(4) Internal damping makes the nonconservative system destabilized as the damping coefficient is enlarged, but external damping has a stabilizing effect on the system.

(5) Dimensionless stability maps of shear-flexible and damped Beck's columns can be constructed using an analytical approach or a FE approach.

(6) As expected, the critical divergence and flutter loads of the undamped/damped Beck's columns decrease considerably with increasing shear parameter $S$.

\section{Competing Interests}

The authors declare that they have no competing interests.

\section{References}

[1] M. Beck, "Die Knicklast des einseitig eingespannten, tangential gedrückten Stabes," Zeitschrift für angewandte Mathematik und Physik, vol. 3, no. 6, pp. 476-477, 1952.

[2] H. Ziegler, Principles of Structural Stability, Blaisdell Publishing Company, Waltham, Mass, USA, 1968.

[3] V. V. Bolotin, Nonconservative Problems of the Theory of Elastic Stability, Pergamon Press, NewYork, NY, USA, 1963.

[4] H. H. E. Leipholz, Stability of Elastic Systems, SijthotI \& Noordhoff, Rijn, The Netherlands, 1980.

[5] B. N. Rao and G. V. Rao, "Stability of a cantilever column under a tip-concentrated subtangential follower force with damping," Journal of Sound and Vibration, vol. 138, no. 2, pp. 341-344, 1990.

[6] R. V. Vitaliani, A. M. Gasparini, and A. V. Saetta, "Finite element solution of the stability problem for nonlinear undamped and damped systems under nonconservative loading," International Journal of Solids and Structures, vol. 34, no. 19, pp. 2497-2516, 1997.

[7] Y. Sugiyama and M. A. Langthjem, "Physical mechanism of the destabilizing effect of damping in continuous nonconservative dissipative systems," International Journal of NonLinear Mechanics, vol. 42, no. 1, pp. 132-145, 2007.

[8] O. N. Kirillov and F. Verhulst, "Paradoxes of dissipationinduced destabilization or who opened Whitney's umbrella?" Zeitschrift für Angewandte Mathematik und Mechanik, vol. 90, no. 6, pp. 462-488, 2010.
[9] A. Luongo and F. Dannibale, "On the destabilizing effect of damping on discrete and continuous circulatory systems," Journal of Sound and Vibration, vol. 333, no. 24, pp. 6723-6741, 2014.

[10] M. A. Langthjem and Y. Sugiyama, "Dynamic stability of columns subjected to follower loads: a survey," Journal of Sound and Vibration, vol. 238, no. 5, pp. 809-851, 2000.

[11] I. Elishakoff, "Controversy associated with the so-called 'follower forces': critical overview," Applied Mechanics Reviews, vol. 58, no. 2, pp. 117-142, 2005.

[12] A. Kounadis and J. T. Katsikadelis, "Shear and rotatory inertia effect on Beck's column," Journal of Sound and Vibration, vol. 49, no. 2, pp. 171-178, 1976.

[13] S. Y. Lee, T. Y. Chen, and W. R. Wang, "Non-conservative instability of a Timoshenko beam subjected to a partially tangential follower force," Journal of Sound and Vibration, vol. 188, no. 1, pp. 25-38, 1995.

[14] V. Sundararamaiah and G. Venkateswara Rao, "Effect of shear deformation and rotatory inertia on the stability of Beck's and Leipholz's columns," AIAA journal, vol. 18, no. 1, pp. 124-125, 1980.

[15] V. Sundararamaiah and G. Venkateswara Rao, "Stability of short Beck and Leipholz columns on elastic foundation," AIAA Journal, vol. 21, no. 7, pp. 1053-1054, 1983.

[16] I. Lottati and I. Elishakoff, "Influence of the shear deformation and rotary inertia on the flutter of a cantilever subjected to a follower force-exact and symbolic manipulation solutions," in Refined Dynamical Theories of Beams, Plates and Shells and Their Applications, pp. 261-273, Springer, Berlin, Germany, 1987.

[17] L.-W. Chen and D.-M. Ku, "Stability analysis of a timoshenko beam subjected to distributed follower forces using finite elements," Computers and Structures, vol. 41, no. 4, pp. 813-819, 1991.

[18] B. J. Ryu, K. Katayama, and Y. Sugiyama, "Dynamic stability of Timoshenko columns subjected to subtangential forces," Computers and Structures, vol. 68, no. 5, pp. 499-512, 1998.

[19] M. M. Attard, J.-S. Lee, and M.-Y. Kim, "Dynamic stability of shear-flexible Beck's columns based on Engesser's and Haringx's buckling theories," Computers and Structures, vol. 86, no. 21-22, pp. 2042-2055, 2008.

[20] V. V. Bolotin and N. I. Zhinzher, "Effects of damping on stability of elastic systems subjected to nonconservative forces," International Journal of Solids and Structures, vol. 5, no. 9, pp. 965-989, 1969.

[21] G. Herrmann and I.-c. Jong, "On the destabilizing effect of damping in nonconservative elastic systems," Journal of Applied Mechanics, vol. 32, no. 3, pp. 592-597, 1965.

[22] O. N. Kirillov and A. P. Seyranian, "The effect of small internal and external damping on the stability of distributed nonconservative systems," Journal of Applied Mathematics and Mechanics, vol. 69, no. 4, pp. 529-552, 2005.

[23] A. Luongo and F. D’Annibale, "Bifurcation analysis of damped visco-elastic planar beams under simultaneous gravitational and follower forces," International Journal of Modern Physics B, vol. 26, no. 25, Article ID 1246015, 2012.

[24] A. Luongo and F. D'Annibale, "Double zero bifurcation of non-linear viscoelastic beams under conservative and non-conservative loads," International Journal of Non-Linear Mechanics, vol. 55, pp. 128-139, 2013.

[25] S. B. Andersen and J. J. Thomsen, "Post-critical behavior of Beck's column with a tip mass," International Journal of NonLinear Mechanics, vol. 37, no. 1, pp. 135-151, 2002. 
[26] J.-S. Lee, N.-I. Kim, and M.-Y. Kim, "Sub-tangentially loaded and damped Beck's columns on two-parameter elastic foundation," Journal of Sound and Vibration, vol. 306, no. 3-5, pp. 766789, 2007.

[27] M.-Y. Kim, J.-S. Lee, and M. M. Attard, "Stability of damped columns on a Winkler foundation under sub-tangential follower forces," International Journal of Structural Stability and Dynamics, vol. 13, no. 2, Article ID 1350020, 27 pages, 2013.

[28] I. Elishakoff, J. Kaplunov, and E. Nolde, "celebrating the centenary of Timoshenko's study of effects of shear deformation and rotary inertia," Applied Mechanics Reviews, vol. 67, no. 6, Article ID 060802, 2015.

[29] F. M. Detinko, "Lumped damping and stability of Beck column with a tip mass," International Journal of Solids and Structures, vol. 40, no. 17, pp. 4479-4486, 2003.

[30] S. Wolfram, Mathematica, Addison-Wesley, Reading, Mass, USA, 2nd edition, 1991. 


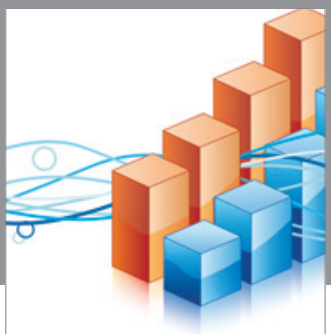

Advances in

Operations Research

vatem alat4

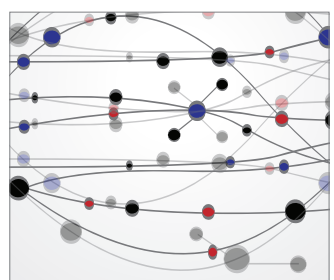

\section{The Scientific} World Journal
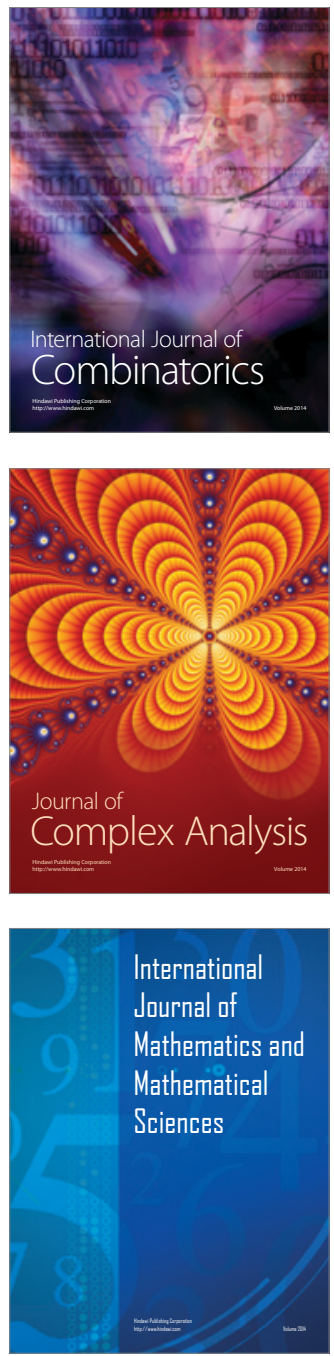
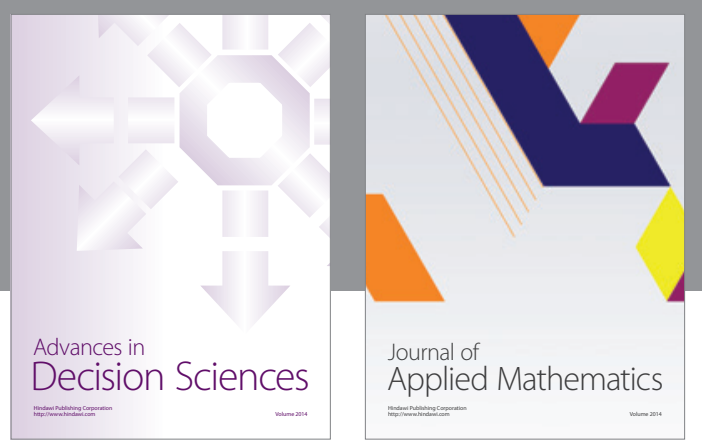

Algebra

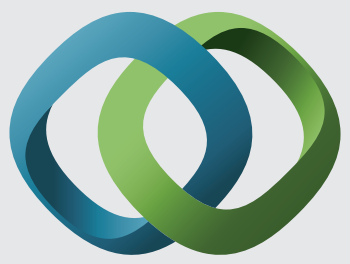

\section{Hindawi}

Submit your manuscripts at

http://www.hindawi.com
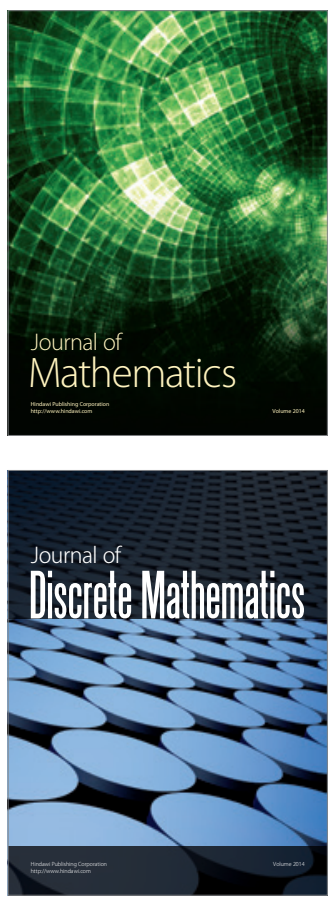

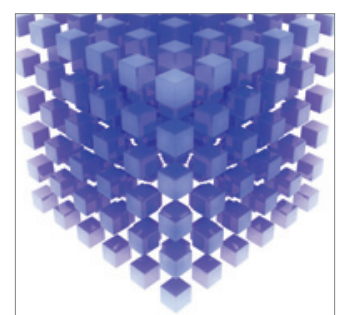

Mathematical Problems in Engineering
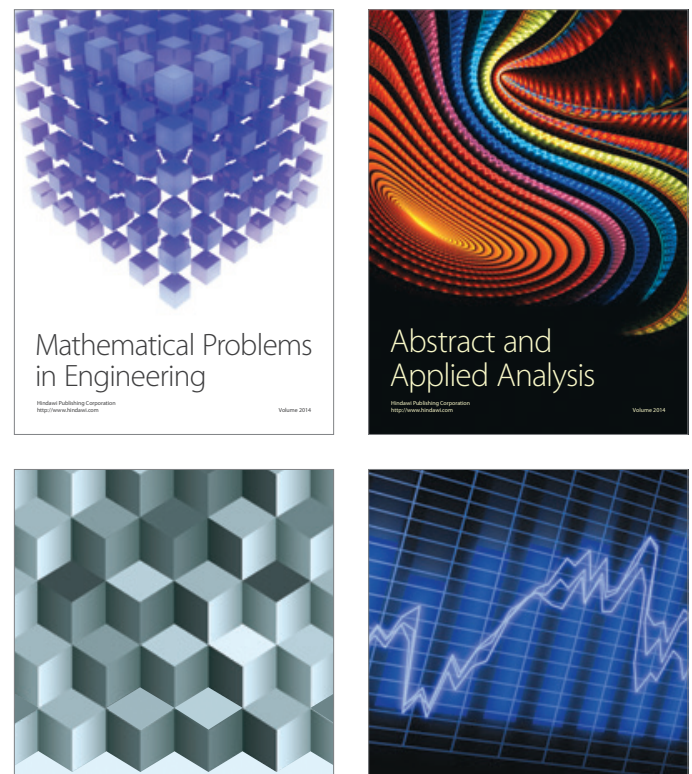

Journal of

Function Spaces

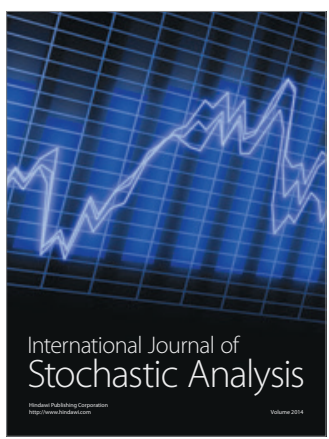

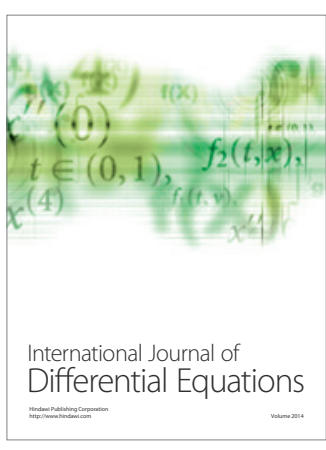
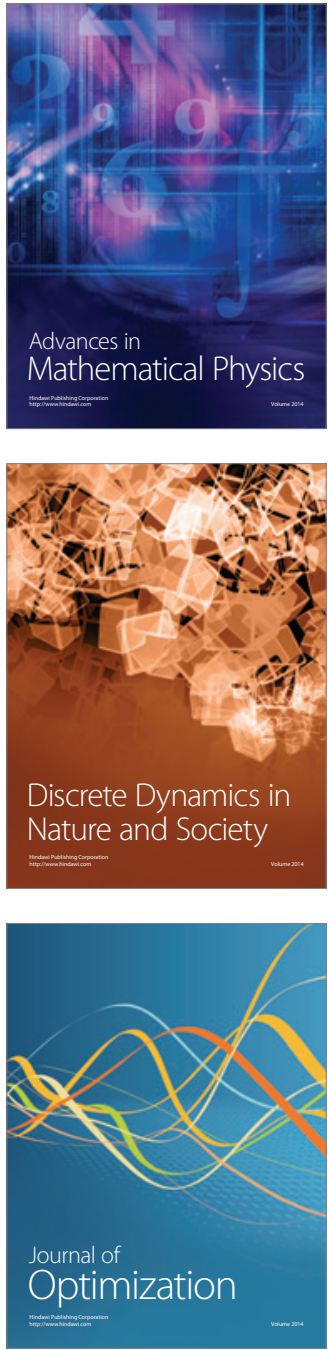